\title{
WEAK SOLUTIONS TO LUBRICATION SYSTEMS DESCRIBING THE EVOLUTION OF BILAYER THIN FILMS*
}

\author{
SEBASTIAN JACHALSKI ${ }^{\dagger}$, GEORGY KITAVTSEV ${ }^{\ddagger}$, AND ROMAN TARANETS ${ }^{\S}$
}

\begin{abstract}
The existence of global non-negative weak solutions is proved for coupled onedimensional lubrication systems that describe the evolution of nanoscopic bilayer thin polymer films that take account of Navier-slip or no-slip conditions at both liquid-liquid and liquid-solid interfaces. In addition, in the presence of attractive van der Waals and repulsive Born intermolecular interactions existence of positive smooth solutions is shown.
\end{abstract}

Key words. Bilayer systems, lubrication equations, weak solutions, slippage.

AMS subject classifications. 35D30, 76A20, 76D08.

\section{Introduction}

During the past few decades lubrication theory was successfully applied to the modelling of dewetting processes in micro and nanoscopic liquid films on solid substrates; see e.g. $[1,2,3,4,5,6,7,8,9]$ to name a few, and for a review we refer to [10] and references therein. A typical closed-form one-dimensional lubrication equation derived from the equations for conservation of mass and momentum, together with boundary conditions for the tangential and normal stresses, as well as the kinematic condition at the free boundary, impermeability and a Navier-slip condition at the liquid-solid interface has the form

$$
\partial_{t} h=-\left(M(h)\left(h_{x x}-\Pi(h)\right)_{x}\right)_{x},
$$

where the function $h(x, t)$ denotes the height profile for the free surface of the film and $\Pi(h)$ the contribution due to intermolecular forces. The mobility function in its most common form reads $M(h)=h^{3}+b h^{2}$, where $b>0$ denotes the slip-length parameter. However, for different magnitudes of $b$ it can also take the form $M(h)=h^{3}$ (no-slip) or $M(h)=b h^{2}$ (intermediate-slip). For a derivation of the non-dimensional equation 1.1 and an overview on the hierarchy of slip models we refer to [5].

Recently, this model was generalized to a coupled system of equations describing morphology and dynamics of a layered system of two viscous, immiscible, nanoscopic Newtonian fluids evolving on a solid substrate [11, 12, 13]. First studies concerning linear stability analysis and numerical simulations for such systems were done in $[11,14,15]$, even in the presence of surfactants [16] or interfacial slip between the solid substrate and the lower liquid [13]. The structure of stationary solutions was considered in $[13,17]$.

Interestingly, slip at liquid-liquid interfaces is rarely discussed in the context of two-layer thin film models. However, motivated by experimental evidence [18, 19, 20] and theoretical derivations [21, 22], slip between the lower and upper liquids was

\footnotetext{
*Received: November 13, 2013; accepted (in revised form): April 9, 2013. Communicated by Andrea Bertozzi.

†Weierstrass Institute, Mohrenstraße 39, 10117 Berlin, Germany (sebastian.jachalski@wiasberlin.de).

${ }^{\ddagger}$ Max Planck Institute for Mathematics in the Sciences, Inselstraße 22, 04103 Leipzig, Germany (georgy.kitavtsev@mis.mpg.de).

$\S$ Institute of Applied Mathematics and Mechanics of the National Academy of Sciences of Ukraine, Roza Luxemburg 74, Donetsk, 83114 Ukraine (taranets_r@yahoo.com).
} 


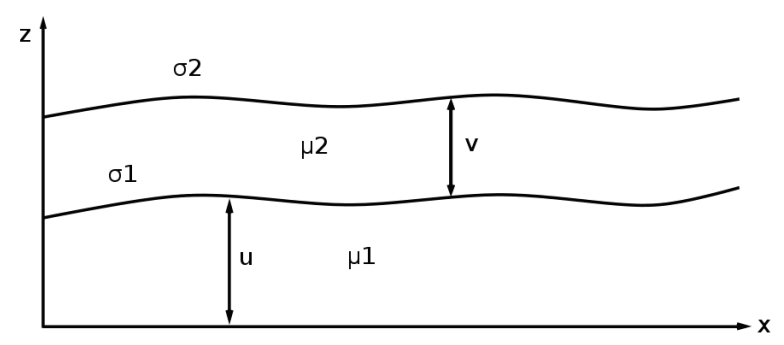

FIG. 1.1. Sketch of a bilayer thin film

incorporated in two-layer lubrication models [12]. In the latter article models for different magnitudes of the slip lengths were derived. Furthermore, linear stability analysis was performed for these models.

Here, we state the system in a form which was derived in [12]:

$$
\begin{aligned}
& u_{t}=\left(M_{11} p_{1, x}+M_{12} p_{2, x}\right)_{x}, \\
& v_{t}=\left(M_{12} p_{1, x}+M_{22} p_{2, x}\right)_{x},
\end{aligned}
$$

where $u(x, t)$ and $v(x, t)$ denote the thicknesses of the lower and upper liquid (see figure 1.1). The pressures $p_{1}(x, t)$ and $p_{2}(x, t)$ are given by

$$
\begin{aligned}
& p_{1}=-(\sigma+1) u_{x x}-v_{x x}+\Pi_{1}(u)+\Pi_{3}(u+v), \\
& p_{2}=-u_{x x}-v_{x x}+\Pi_{2}(v)+\Pi_{3}(u+v) .
\end{aligned}
$$

Already, in [15] it was pointed out that (1.2), (1.3) has a gradient dynamics form. In particular, the pressures are variations of the functional

$$
E(u, v)=\int_{\Omega}\left[\frac{\sigma}{2} u_{x}^{2}+\frac{1}{2}\left(u_{x}+v_{x}\right)^{2}+U_{1}(u)+U_{2}(v)+U_{3}(u+v)\right] d x,
$$

i.e. $p_{1}=\delta E / \delta u, p_{2}=\delta E / \delta v$. The quantities $u_{x x}$ and $v_{x x}$ are the mean curvatures in long-wave approximation and potentials $U_{1}(u), U_{2}(v)$, and $U_{3}(u+v)$ describe the intermolecular interactions between the solid surface, lower liquid, and upper liquid. The influence of these interactions is typically due to the competition between longrange attractive van der Waals and short-range repulsive Born forces; see [6, 23, 24]. In this article we consider two cases: the absence of intermolecular interactions, i.e. $U_{k}(s) \equiv 0$ for $k=1,2,3$, and the case when the van der Waals intermolecular potentials are presented in the form

$$
U_{k}(s)=-\int_{s}^{\infty} \Pi_{k}(\tau) d \tau, \quad \text { with } \quad \Pi_{k}(s)=\frac{1}{s^{n}}-\frac{\gamma_{k}}{s^{m}}\left(n<m, 0<\gamma_{1}, \gamma_{2} \ll 1\right) .
$$

A typical choice for $(n, m)$ is $(3,9)$ corresponding to the standard Lennard-Jones potential.

Considering Navier-slip conditions at the liquid-liquid and liquid-solid interfaces, the mobility matrix takes the form

$$
M=\frac{1}{\mu}\left(\begin{array}{cc}
\frac{1}{3} u^{3}+b_{1} u^{2} & \frac{1}{2} u^{2} v+b_{1} u v \\
\frac{1}{2} u^{2} v+b_{1} u v & \frac{\mu}{3} v^{3}+u v^{2}+b_{1} v^{2}+b(\mu+1) v^{2}
\end{array}\right),
$$


where $b_{1}>0$ and $b \geq 0$ denote the slip lengths at the solid-liquid and liquid-liquid interfaces, respectively. The model parameters $\sigma=\sigma_{1} / \sigma_{2}$ and $\mu=\mu_{1} / \mu_{2}$ in (1.3), (1.4), and (1.6) are positive constants which denote the ratios of surface tensions and viscosities. System (1.2) with mobility matrix (1.6) is called a weak-slip model for two-layer thin films.

Analogously to the case of a single liquid layer on a solid substrate, the mobility matrix depends on the magnitude of the slip lengths. In the case of vanishing slip lengths at the two interfaces (no-slip case), it has the form

$$
M=\frac{1}{\mu}\left(\begin{array}{cc}
\frac{1}{3} u^{3} & \frac{1}{2} u^{2} v \\
\frac{1}{2} u^{2} v & \frac{\mu}{3} v^{3}+u v^{2}
\end{array}\right) .
$$

If the slip lengths $b_{1}$ and $b$ are asymptotically large compared to $u$ and $v$ the corresponding mobility matrix can be stated in the form

$$
M=\frac{1}{\mu}\left(\begin{array}{cc}
b_{1} u^{2} & b_{1} u v \\
b_{1} u v & b_{1} v^{2}+b(\mu+1) v^{2}
\end{array}\right) .
$$

Note that rescaling time by $b_{1}$ and introducing the parameter $\beta:=\frac{b}{b_{1}}(\mu+1)>0$, the latter matrix can be written in the form

$$
M=\frac{1}{\mu}\left(\begin{array}{cc}
u^{2} & u v \\
u v & (1+\beta) v^{2}
\end{array}\right) .
$$

This matrix corresponds to the case of intermediate-slip.

In this study we consider the system (1.2) on a time-space domain $Q_{T}=\Omega \times(0, T)$, where $\Omega=(0,1)$, with boundary conditions

$$
u_{x}=u_{x x x}=v_{x}=v_{x x x}=0 \quad \text { for } x \in \partial \Omega
$$

and the initial functions

$$
u(x, 0)=u_{0}(x) \geq 0, v(x, 0)=v_{0}(x) \geq 0, \quad u_{0}, v_{0} \in H^{1}(\Omega) .
$$

Starting from the seminal work of Bernis and Friedman [25] existence theory of non-negative weak solutions for single non-linear parabolic equations in the form (1.1) was successfully developed (see e.g. [2, 26] and references therein). Note that the system (1.2) inherits from the one-layer lubrication equation 1.1 the high order and degeneracy as one of the fluid heights $u$ or $v$ goes to zero. In contrast to (1.1) there are only few analytical results known about the system (1.2). Existence of nonnegative weak solutions to (1.2) in the no-slip case, i.e. with mobility matrix given by (1.7) was shown recently in [27] using a finite-element approximation under a strong assumption on presence of intermolecular potentials of the form (1.5) between liquid films and between each film and the substrate as well.

In this article, we show existence of non-negative weak solutions to (1.2) with (1.9)-(1.10) for both no-slip (1.7) and intermediate-slip cases (1.8) in the absence of intermolecular forces. In turn, in the presence of intermolecular forces we show that the observed weak solution becomes positive and smooth. 
In our approach we extend ideas introduced in [25] for the single lubrication equation of the form (1.1) to the system (1.2). However, the extension is not straightforward. There are new challenges since the mobility matrix degenerates in more than one way. Beside the case that $u$ and $v$ vanish simultaneously, also the mobility matrix $M$ may degenerates if either $u$ or $v$ becomes zero while the other does not. All these cases have to be treated very carefully. In Section 2 we introduce the corresponding regularized version of the system (1.2) for the no-slip case, with mobility matrix (1.7). By deriving the energy dissipation and corresponding a priori estimates, using the theory of uniformly parabolic systems (see [28]), we show global existence of smooth solutions to the regularized problem. Furthermore, we show that the latter converges to a suitably defined weak solution of the original system (1.2). In Section 3 we prove non-negativity of thus obtained weak solutions in the case when intermolecular forces are absent. Our approach for proving the non-negativity is based on a definition of suitable analogues of Bernis and Friedman entropies for functions $u$ and $v$ and showing their combined dissipation. Moreover, in Section 4 we show global existence of non-negative weak solutions to (1.2) in the intermediate-slip case (1.8).

The results obtained in Section 2 are independent of the presence of intermolecular forces in the equations. In this case we use the properties of the potentials $U_{1}$ and $U_{2}$ given in (1.5), e.g. that they are bounded from below, to show that the weak solutions are positive and smooth (Section 3).

We should point out that an alternative proof for the existence of weak solutions in the no-slip case considered without intermolecular forces appeared in parallel to our article in [29]. In the Section 5 we discuss our results and, in particular, compare them with those of [29].

\section{Existence of weak solutions in the no-slip case}

In this section we consider the system (1.2) without intermolecular interactions, i.e. the pressures $p_{1}, p_{2}$ in (1.3) are given by

$$
p_{1}=-(\sigma+1) u_{x x}-v_{x x}, \quad p_{2}=-u_{x x}-v_{x x} ;
$$

and with the no-slip mobility matrix

$$
M=\frac{1}{\mu}\left(\begin{array}{cc}
\frac{1}{3}|u|^{3} & \frac{1}{2} u^{2} v \\
\frac{1}{2} u^{2} v & \frac{\mu}{3}|v|^{3}+|u| v^{2}
\end{array}\right) .
$$

Notice that we replaced some $u$ and $v$ on the main diagonal in the mobility matrix (1.7) by their absolute values to ensure that the latter is positive semi-definite. We will prove existence of global weak solutions to (1.2), (2.1) considered with boundary and initial conditions (1.9)-(1.10). We begin our analysis with the introduction of a regularized version of (1.2), (1.10) and a derivation of a priori estimates for its solutions.

2.1. Regularised system and a priori estimates. Since (1.2) is degenerate at $u=0$ or $v=0$, we approximate it by a family of non-degenerate equations

$$
\begin{aligned}
& u_{t}-\left(\left(M_{11}+\varepsilon\right) p_{1, x}+M_{12} p_{2, x}\right)_{x}=0, \quad \text { in } Q_{T}, \\
& v_{t}-\left(M_{21} p_{1, x}+\left(M_{22}+\varepsilon\right) p_{2, x}\right)_{x}=0,
\end{aligned}
$$

where $\varepsilon>0$ is arbitrary. Note that the regularized mobility matrix is positive definite for all $u$ and $v$. Correspondingly the system (2.2) is uniformly parabolic in the 
Petrovskii sense (see [28] for the definition). Furthermore, we approximate $u_{0}$ and $v_{0}$ in the $H^{1}(\Omega)$-norm by $C^{4+\alpha}$ functions $u_{0 \varepsilon}$ and $v_{0 \varepsilon}$ satisfying (1.9),

$$
u_{0 \varepsilon}(x) \geq u_{0}(x) \text { and } v_{0 \varepsilon}(x) \geq v_{0}(x) \text { for } x \in \bar{\Omega},
$$

and replace (1.10) by

$$
u(x, 0)=u_{0 \varepsilon}(x), v(x, 0)=v_{0 \varepsilon}(x) .
$$

By [28, Theorem. 6.3, p. 302] the system (2.2) considered with (1.9), (2.4) has a unique local solution $\left(u_{\varepsilon}, v_{\varepsilon}\right)$ in $Q_{\tau}$ for some small $\tau=\tau(\varepsilon)>0$.

Everywhere below in this article we will denote by $C$ positive constants independent of $\varepsilon$ which may vary from line to line. Let us also introduce notations

$$
M_{\varepsilon}:=M\left(u_{\varepsilon}, v_{\varepsilon}\right), \quad p_{1, \varepsilon}:=-(\sigma+1) u_{\varepsilon, x x}-v_{\varepsilon, x x}, \text { and } p_{2, \varepsilon}:=-u_{\varepsilon, x x}-v_{\varepsilon, x x} .
$$

A priori estimates. Let us recall the energy (Lyapunov) functional (1.4) for the system (1.2) coupled with (1.9) without contributions of intermolecular interaction:

$$
E\left(u_{\varepsilon}, v_{\varepsilon}\right):=\frac{1}{2} \int_{\Omega}\left[\sigma u_{\varepsilon, x}^{2}+\left(u_{\varepsilon, x}+v_{\varepsilon, x}\right)^{2}\right] d x
$$

Indeed, differentiating the latter in time along solutions of (1.2) with (1.9) one obtains the corresponding energy equality

$$
\frac{d}{d t} E\left(u_{\varepsilon}, v_{\varepsilon}\right)+\int_{\Omega}\left(M_{11 \varepsilon} p_{1 \varepsilon, x}^{2}+2 M_{12 \varepsilon} p_{1 \varepsilon, x} p_{2 \varepsilon, x}+M_{22 \varepsilon} p_{2 \varepsilon, x}^{2}\right) d x+\varepsilon \int_{\Omega}\left(p_{1 \varepsilon, x}^{2}+p_{2 \varepsilon, x}^{2}\right) d x=0 .
$$

Note that the second term in (2.7) is non-negative since $M_{\varepsilon}$ is positive semi-definite. By the approximation properties of $u_{0 \varepsilon}, v_{0 \varepsilon}$ one has

$$
\begin{aligned}
& \int_{\Omega} u_{0 \varepsilon, x}^{2} d x \leq(1+\eta(\varepsilon)) \int_{\Omega} u_{0, x}^{2} d x, \\
& \int_{\Omega} v_{0 \varepsilon, x}^{2} d x \leq(1+\eta(\varepsilon)) \int_{\Omega} v_{0, x}^{2} d x,
\end{aligned}
$$

where $\eta(\varepsilon) \rightarrow 0$ if $\varepsilon \rightarrow 0$ and therefore $E\left(u_{0 \varepsilon}, v_{0 \varepsilon}\right) \leq C$ holds. This together with (2.7) implies the following a priori estimates:

$$
\sup _{t \in(0, \tau)} \int_{\Omega} u_{\varepsilon, x}^{2} d x \leq C, \sup _{t \in(0, \tau)} \int_{\Omega} v_{\varepsilon, x}^{2} d x \leq C,
$$

and

$$
\begin{array}{r}
\iint_{Q_{\tau}}\left(M_{11 \varepsilon} p_{1 \varepsilon, x}^{2}+2 M_{12 \varepsilon} p_{1 \varepsilon, x} p_{2 \varepsilon, x}+M_{22 \varepsilon} p_{2 \varepsilon, x}^{2}\right) d x \leq C, \\
\varepsilon \iint_{Q_{\tau}}\left(p_{1 \varepsilon, x}^{2}+p_{2 \varepsilon, x}^{2}\right) d x \leq C .
\end{array}
$$


Integrating (2.2) in time we deduce the conservation of mass law

$$
\int_{\Omega} u_{\varepsilon}(x, t) d x=\int_{\Omega} u_{0 \varepsilon}(x) d x, \int_{\Omega} v_{\varepsilon}(x, t) d x=\int_{\Omega} v_{0 \varepsilon}(x) d x
$$

for all $t \in(0, \tau)$. Using this, (2.8), Poincaré's inequality, and the Sobolev embedding theorem

$$
H^{1}(\Omega) \subset C^{\frac{1}{2}}(\bar{\Omega})
$$

one obtains

$$
\left\|u_{\varepsilon}(., t)\right\|_{C^{\frac{1}{2}(\bar{\Omega})}} \leq C,\left\|v_{\varepsilon}(., t)\right\|_{C^{\frac{1}{2}(\bar{\Omega})}} \leq C .
$$

Next, we obtain uniform Hölder estimates for $u_{\varepsilon}$ and $v_{\varepsilon}$ in time. Let us introduce the fluxes

$$
J_{1, \varepsilon}=-M_{11 \varepsilon} p_{1 \varepsilon, x}-M_{12 \varepsilon} p_{2 \varepsilon, x} \text { and } \quad J_{2, \varepsilon}=-M_{21 \varepsilon} p_{1 \varepsilon, x}-M_{22 \varepsilon} p_{2 \varepsilon, x} .
$$

Observe that for every $t \in(0, \tau)$

$$
\begin{aligned}
\iint_{Q_{t}} J_{1, \varepsilon}^{2} d x d t & \leq C \iint_{Q_{t}} M_{11 \varepsilon}\left(M_{11 \varepsilon} p_{1 \varepsilon, x}^{2}+2 M_{12 \varepsilon} p_{1 \varepsilon, x} p_{2 \varepsilon, x}+M_{22 \varepsilon} p_{2 \varepsilon, x}^{2}\right) d x d t \\
& \leq C,
\end{aligned}
$$

where we use $M_{12 \varepsilon}^{2} \leq M_{11 \varepsilon} M_{22 \varepsilon},(2.10)$, and (2.13). Analogously,

$$
\iint_{Q_{t}} J_{2, \varepsilon}^{2} d x d t \leq C
$$

holds. Again, from (2.2) we derive by two partial integrations the relations

$$
\iint_{Q_{t}} u_{\varepsilon} \phi_{t}=-\iint_{Q_{t}} J_{1, \varepsilon} \phi_{x} \text { and } \iint_{Q_{t}} v_{\varepsilon} \phi_{t}=-\iint_{Q_{t}} J_{2, \varepsilon} \phi_{x} .
$$

Now, using (2.14)-(2.15) and (2.16), considered with the special test function $\phi$ taken exactly as in the analogous proof for the single layer lubrication equation 1.1 in [25, Lemma 2.1], one obtains that for all $x \in \bar{\Omega}$ and $t_{1}, t_{2}$ in $(0, \tau)$ the following holds:

$$
\begin{aligned}
\left|u_{\varepsilon}\left(x, t_{2}\right)-u_{\varepsilon}\left(x, t_{1}\right)\right| \leq C\left|t_{2}-t_{1}\right|^{\frac{1}{8}}, \\
\left|v_{\varepsilon}\left(x, t_{2}\right)-v_{\varepsilon}\left(x, t_{1}\right)\right| \leq C\left|t_{2}-t_{1}\right|^{\frac{1}{8}} .
\end{aligned}
$$

Conclusion. The relations (2.13) and (2.17) imply upper bounds on the $C_{x, t}^{\frac{1}{2}, \frac{1}{8}}$ norms of $u_{\varepsilon}$ and $v_{\varepsilon}$ in $Q_{\tau}$, which are independent of $\tau$ and $\varepsilon$. These a priori bounds allow us to conclude that $\left(u_{\varepsilon}, v_{\varepsilon}\right)$ can be extended step-by-step to a solution of (2.2) considered with (1.9), (2.4) in $Q_{T}$ for any positive $T>0$ (see [28, Theorem. 9.3, p. $316])$, and that

the sequences $\left\{u_{\varepsilon}\right\}_{\varepsilon>0}$ and $\left\{v_{\varepsilon}\right\}_{\varepsilon>0}$ are uniformly bounded and equicontinuous families in $\bar{Q}_{T}$. 
2.2. Convergence to global weak solutions. Here, we show that solution $\left(u_{\varepsilon}, v_{\varepsilon}\right)$ of the regularized system $(2.2)$ converges to a suitably defined global weak solution of the initial system (1.2). By (2.18), every sequence $\varepsilon \longrightarrow 0$ has a subsequence (for brevity neither are labeled) such that

$$
u_{\varepsilon} \longrightarrow u, v_{\varepsilon} \longrightarrow v \quad \text { uniformly in } \bar{Q}_{T} \text {. }
$$

Note that, due to the uniform bounds (2.13) and (2.17), any such limit $(u, v)$ can be defined globally in time using a standard Cantor diagonal argument (choosing a sequence $\left.T_{n} \rightarrow \infty\right)$.

Theorem 2.1. Any pair $(u, v)$ obtained as in (2.19) satisfies for any $T>0$ the following properties:

$$
\begin{aligned}
& (u, v) \in C_{x, t}^{1 / 2,1 / 8}\left(\bar{Q}_{T}\right) \times C_{x, t}^{1 / 2,1 / 8}\left(\bar{Q}_{T}\right),(u, v) \in C_{x, t}^{4,1}(P) \times C_{x, t}^{4,1}(P), \\
& M_{11} p_{1, x}+M_{12} p_{2, x}, M_{21} p_{1, x}+M_{22} p_{2, x} \in L^{2}(P), \\
& |u|^{3} p_{1, x} \in L^{2}(R),|v|^{3} p_{2, x} \in L^{2}(S),
\end{aligned}
$$

where $P=\bar{Q}_{T} \backslash(\{u=0\} \cup\{v=0\} \cup\{t=0\}), R=\bar{Q}_{T} \cap\{v=0\} \cap\{|u|>0\}$, and $S=\bar{Q}_{T} \cap$ $\{u=0\} \cap\{|v|>0\}$. Furthermore, there exists a function $w \in L^{2}(R)$, such that $(u, v)$ satisfies (1.2) in the following sense:

$$
\begin{aligned}
& \iint_{Q_{T}} u \phi_{t}-\iint_{P}\left(M_{11} p_{1, x}+M_{12} p_{2, x}\right) \phi_{x}-\iint_{R}\left(\frac{1}{3 \mu}|u|^{3} p_{1, x}+\frac{1}{2 \mu} u^{2} w\right) \phi_{x}=0, \\
& \iint_{Q_{T}} v \phi_{t}-\iint_{P}\left(M_{21} p_{1, x}+M_{22} p_{2, x}\right) \phi_{x}-\iint_{S} \frac{1}{3}|v|^{3} p_{2, x} \phi_{x}=0,
\end{aligned}
$$

for all $\phi \in \operatorname{Lip}\left(\bar{Q}_{T}\right), \phi=0$ near $t=0$ and $t=T$;

$$
\begin{aligned}
& u(x, 0)=u_{0}(x), v(x, 0)=v_{0}(x), \quad x \in \bar{\Omega}, \\
& \int_{\Omega} u(x, t) d x=\left\|u_{0}\right\|_{L^{1}(\Omega)}, \int_{\Omega} v(x, t) d x=\left\|v_{0}\right\|_{L^{1}(\Omega)}, \\
& u_{x}(\cdot, t) \rightarrow u_{0, x}(\cdot) \text { and } v_{x}(\cdot, t) \rightarrow v_{0, x}(\cdot) \text { strongly in } L^{2}(\Omega) \text { as } t \rightarrow 0,
\end{aligned}
$$

and

$u$ and $v$ satisfy (1.9) at all points of the lateral

$$
\text { boundary, where } u \neq 0 \text { and } v \neq 0 \text {. }
$$

Finally, the following energy inequality holds:

$$
E(u(\cdot, T), v(\cdot, T))+\iint_{P}\left(M_{11} p_{1, x}^{2}+2 M_{12} p_{1, x} p_{2, x}+M_{22} p_{2, x}^{2}\right) \leq E\left(u_{0}, v_{0}\right) .
$$

Proof. By the properties of the constructed solutions $\left(u_{\varepsilon}, v_{\varepsilon}\right)$ to the regularized system and their uniform convergence to $(u, v)$, the first assertion in $(2.20)$ and also (2.25)-(2.26) follow immediately. Using (2.10), we observe that 


$$
\begin{aligned}
\iint_{Q_{T}}\left(M_{11 \varepsilon} p_{1, \varepsilon, x}^{2}+M_{22 \varepsilon} p_{2 \varepsilon, x}^{2}\right) d x d t & \leq C-2 \iint_{Q_{T}} M_{12 \varepsilon} p_{1 \varepsilon, x} p_{2 \varepsilon, x} d x d t \\
& =C-\frac{1}{\mu} \iint_{Q_{T}} u_{\varepsilon}^{2} v_{\varepsilon} p_{1 \varepsilon, x} p_{2 \varepsilon, x} d x d t .
\end{aligned}
$$

From this, applying Young's inequality,

$$
\left|u_{\varepsilon}^{2} v_{\varepsilon} p_{1 \varepsilon, x} p_{2 \varepsilon, x}\right| \leq \frac{7}{24}\left|u_{\varepsilon}\right|^{3} p_{1 \varepsilon, x}^{2}+\frac{6}{7}\left|u_{\varepsilon}\right|\left|v_{\varepsilon}\right|^{2} p_{2 \varepsilon, x}^{2}
$$

and one obtains

$$
\iint_{Q_{T}}\left(M_{11 \varepsilon} p_{1, \varepsilon, x}^{2}+M_{22 \varepsilon} p_{2 \varepsilon, x}^{2}\right) d x d t \leq C,
$$

and therefore estimates

$$
\iint_{Q_{T}}\left|u_{\varepsilon}\right|^{3} p_{1 \varepsilon, x}^{2} d x d t \leq C, \iint_{Q_{T}}\left|v_{\varepsilon}\right|^{3} p_{2 \varepsilon, x}^{2} d x d t \leq C, \iint_{Q_{T}}\left|u_{\varepsilon}\right| v_{\varepsilon}^{2} p_{2 \varepsilon, x}^{2} d x d t \leq C .
$$

For any $\phi$ as in $(2.23)$ one has

$$
\begin{aligned}
& \iint_{Q_{T}} u_{\varepsilon} \phi_{t} d x d t-\iint_{Q_{T}}\left(\left(M_{11 \varepsilon}+\varepsilon\right) p_{1 \varepsilon, x}+M_{12 \varepsilon} p_{2 \varepsilon, x}\right) \phi_{x} d x d t=0, \\
& \iint_{Q_{T}} v_{\varepsilon} \phi_{t} d x d t-\iint_{Q_{T}}\left(M_{21 \varepsilon} p_{1 \varepsilon, x}+\left(M_{22 \varepsilon}+\varepsilon\right) p_{2 \varepsilon, x}\right) \phi_{x} d x d t=0 .
\end{aligned}
$$

By (2.14) and (2.15) there exist $J_{1}, J_{2} \in L^{2}\left(Q_{T}\right)$ and a subsequence as $\varepsilon \rightarrow 0$ such that

$$
\left(-M_{11 \varepsilon} p_{1 \varepsilon, x}-M_{12 \varepsilon} p_{2 \varepsilon, x}\right) \rightarrow J_{1}
$$

and $\left(-M_{21 \varepsilon} p_{1 \varepsilon, x}-M_{22 \varepsilon} p_{2 \varepsilon, x}\right) \rightarrow J_{2}$ weakly in $L^{2}\left(Q_{T}\right)$.

Additionally, by (2.11),

$$
\varepsilon \iint_{Q_{T}} p_{1 \varepsilon, x} \phi_{x} d x d t \rightarrow 0, \varepsilon \iint_{Q_{T}} p_{2 \varepsilon, x} \phi_{x} d x d t \rightarrow 0 \text { as } \varepsilon \rightarrow 0 .
$$

By the regularity theory of uniformly parabolic systems and the uniform Hölder continuity of the $u_{\varepsilon}$ and $v_{\varepsilon}$ we deduce that $(u, v) \in C_{x, t}^{4,1}(P) \times C_{x, t}^{4,1}(P)$ and

$$
J_{1}=-M_{11} p_{1, x}-M_{12} p_{2, x}, J_{2}=-M_{21} p_{1, x}-M_{22} p_{2, x} \quad \text { on } P .
$$

Next, for a fixed $\delta>0$ define a set $I_{1, \delta}=\{|v| \leq \delta<|u|\}$. From the estimates (2.31) it follows that there exists $w \in L^{2}\left(I_{1, \delta}\right)$ such that $p_{1 \varepsilon, x} \rightarrow p_{1, x}$ and $v_{\varepsilon} p_{2 \varepsilon, x} \rightarrow w$ weakly in $L^{2}\left(I_{1, \delta}\right)$ as $\varepsilon \rightarrow 0$. Therefore, one obtains

$$
\iint_{I_{1, \delta}}\left(M_{11 \varepsilon} p_{1 \varepsilon, x}+M_{12 \varepsilon} p_{2 \varepsilon, x}\right) \phi_{x} d x d t \rightarrow \iint_{I_{1, \delta}}\left(\frac{1}{3 \mu}|u|^{3} p_{1, x}+\frac{1}{2 \mu} u u^{2} w\right) \phi_{x} d x d t
$$


as $\varepsilon \rightarrow 0$. On the other hand, one has the estimate

$$
\begin{aligned}
& \iint_{|u| \leq \delta}\left(M_{11 \varepsilon} p_{1 \varepsilon, x}+M_{12 \varepsilon} p_{2 \varepsilon, x}\right) \phi_{x} d x d t \\
\leq & C\left(\iint_{|u| \leq \delta} M_{11 \varepsilon}\left(M_{11 \varepsilon} p_{1 \varepsilon, x}^{2}+2 M_{12 \varepsilon} p_{1 \varepsilon, x} p_{2 \varepsilon, x}+M_{22 \varepsilon} p_{2 \varepsilon, x}^{2}\right) d x d t\right)^{1 / 2} \\
\leq & C \delta^{3 / 2} .
\end{aligned}
$$

Let us decompose the second term in (2.32) as

$$
\begin{array}{r}
\iint_{|u|>\delta,|v|>\delta}\left(M_{11 \varepsilon} p_{1 \varepsilon, x}+M_{12 \varepsilon} p_{2 \varepsilon, x}\right) \phi_{x} d x d t+\iint_{|u| \leq \delta}\left(M_{11 \varepsilon} p_{1 \varepsilon, x}+M_{12 \varepsilon} p_{2 \varepsilon, x}\right) \phi_{x} d x d t \\
+\varepsilon \iint_{Q_{T}} p_{1 \varepsilon, x} \phi_{x} d x d t+\iint_{|u|>\delta,|v| \leq \delta}\left(M_{11 \varepsilon} p_{1 \varepsilon, x}+M_{12 \varepsilon} p_{2 \varepsilon, x}\right) \phi_{x} d x d t
\end{array}
$$

and take the limit $\delta \rightarrow 0$ extracting a proper diagonal subsequence $\varepsilon \rightarrow 0$ as follows. Using (2.35) one has

$$
\begin{aligned}
& \lim _{\delta \rightarrow 0}\left|\int_{|u|>\delta,|v|>\delta}\left(M_{11 \varepsilon} p_{1 \varepsilon, x}+M_{12 \varepsilon} p_{2 \varepsilon, x}\right) \phi_{x} d x d t+\iint_{P} J_{1} \phi_{x} d x d t\right| \\
& \leq \lim _{\delta \rightarrow 0}\left|\iint_{|u|>\delta,|v|>\delta}\left(M_{11 \varepsilon} p_{1 \varepsilon, x}+M_{12 \varepsilon} p_{2 \varepsilon, x}\right) \phi_{x} d x d t+\iint_{|u|>\delta,|v|>\delta} J_{1} \phi_{x} d x d t\right| \\
& +\lim _{\delta \rightarrow 0}\left|\iint_{P} J_{1} \phi_{x} d x d t-\iint_{|u|>\delta,|v|>\delta} J_{1} \phi_{x} d x d t\right|=0,
\end{aligned}
$$

where in the last line we used that $J_{1} \phi_{x}$ is a bounded continuous function in $P$. Furthermore, from (2.36) one obtains

$$
\begin{gathered}
\lim _{\delta \rightarrow 0}\left|\iint_{I_{1, \delta}}\left(M_{11 \varepsilon} p_{1 \varepsilon, x}+M_{12 \varepsilon} p_{2 \varepsilon, x}\right) \phi_{x} d x d t-\iint_{v=0,|u|>0}\left(\frac{1}{3 \mu}|u|^{3} p_{1, x}+\frac{1}{2 \mu} u^{2} w\right) \phi_{x} d x d t\right| \\
\leq \lim _{\delta \rightarrow 0}\left|\iint_{I_{1, \delta}}\left(M_{11 \varepsilon} p_{1 \varepsilon, x}+M_{12 \varepsilon} p_{2 \varepsilon, x}\right) \phi_{x} d x d t-\iint_{I_{1, \delta}}\left(\frac{1}{3 \mu}|u|^{3} p_{1, x}+\frac{1}{2 \mu} u^{2} w\right) \phi_{x} d x d t\right| \\
+\lim _{\delta \rightarrow 0} \mid \int_{|v| \leq \delta,|u|>\delta}\left(\frac{1}{3 \mu}|u|^{3} p_{1, x}+\frac{1}{2 \mu} u^{2} w\right) \phi_{x} d x d t \\
\quad-\iint_{v=0,|u|>0}\left(\frac{1}{3 \mu}|u|^{3} p_{1, x}+\frac{1}{2 \mu} u^{2} w\right) \phi_{x} d x d t \mid \\
\leq \lim _{\delta \rightarrow 0}\left|\iint_{\delta \geq|v|}\left(\frac{1}{3 \mu}|u|^{3} p_{1, x}+\frac{1}{2 \mu} u^{2} w\right) \phi_{x} d x d t\right|
\end{gathered}
$$




$$
\begin{aligned}
& \leq \lim _{\delta \rightarrow 0} C\left[\left(\iint_{\delta \geq|v|}|u|^{3} d x d t \iint_{\delta \geq|v|}|u|^{3} p_{1, x}^{2} d x d t\right)^{\frac{1}{2}}+\left(\iint_{\delta \geq|v|}|u|^{4} d x d t \iint_{\delta \geq|v|} w^{2} d x d t\right)^{\frac{1}{2}}\right] \\
& \leq \lim _{\delta \rightarrow 0} C\left(\iint_{\delta \geq|v|} 1 d x d t\right)^{\frac{1}{2}}=0
\end{aligned}
$$

where in the last inequality we used (2.20) and (2.31). Therefore, the last two estimates together with (2.37) imply that there exists a subsequence $\varepsilon \rightarrow 0$ such that (2.32) converges to (2.23).

Similarly, defining for a fixed $\delta \geq 0$ the set $I_{2, \delta}=\{|u| \leq \delta<|v|\}$ one can estimate

$$
\iint_{|v| \leq \delta}\left(M_{21 \varepsilon} p_{1 \varepsilon, x}+M_{22 \varepsilon} p_{2 \varepsilon, x}\right) \phi_{x} d x d t \leq C \delta,
$$

and

$$
\begin{aligned}
& \iint_{I_{2, \delta}}\left(M_{21 \varepsilon} p_{1 \varepsilon, x}+M_{22 \varepsilon} p_{2 \varepsilon, x}-\frac{1}{3}\left|v_{\varepsilon}\right|^{3} p_{2 \varepsilon, x}\right) \phi_{x} d x d t \\
& \leq C\left(\int \int _ { I _ { 2 , \delta } } \left(M_{21 \varepsilon}^{2} p_{1 \varepsilon, x}^{2}+2 M_{21 \varepsilon}\left(M_{22 \varepsilon}-\frac{1}{3}\left|v_{\varepsilon}\right|^{3}\right) p_{1 \varepsilon, x} p_{2 \varepsilon, x}\right.\right. \\
& \left.\left.\quad+\left(M_{22 \varepsilon}-\frac{1}{3}\left|v_{\varepsilon}\right|^{3}\right)^{2} p_{2 \varepsilon, x}^{2}\right) d x d t\right)^{1 / 2} \\
& \leq C\left(\iint_{I_{2, \delta}} \frac{1}{\mu}\left|u_{\varepsilon}\right| v_{\varepsilon}^{2}\left(M_{11 \varepsilon} p_{1 \varepsilon, x}^{2}+2 M_{21 \varepsilon} p_{1 \varepsilon, x} p_{2 \varepsilon, x}+M_{22 \varepsilon} p_{2 \varepsilon, x}^{2}\right) d x d t\right)^{1 / 2} \\
& \leq C \delta^{1 / 2} .
\end{aligned}
$$

Moreover, again from (2.31) it follows that $p_{2 \varepsilon, x} \rightarrow p_{2, x}$ weakly in $L^{2}\left(I_{2, \delta}\right)$ as $\varepsilon \rightarrow 0$, and therefore we deduce that

$$
\iint_{I_{2, \delta}}\left(\frac{1}{3}\left|v_{\varepsilon}\right|^{3} p_{2 \varepsilon, x}\right) \phi_{x} d x d t \rightarrow \iint_{I_{2, \delta}}\left(\frac{1}{3}|v|^{3} p_{2, x}\right) \phi_{x} d x d t
$$

as $\varepsilon \rightarrow 0$. Taking the limit $\delta \rightarrow 0$ and the corresponding diagonal sequence $\varepsilon \rightarrow 0$ in (2.33) (as was done before for 2.32) shows that it converges to (2.24) in view of (2.39)-(2.41).

To prove (2.27), notice that from $u_{0, \varepsilon} \rightarrow u_{0}, v_{0, \varepsilon} \rightarrow v_{0}$ in $H^{1}(\Omega)$ and (2.7) we get

$$
\limsup _{t \rightarrow 0} \int_{\Omega}\left(\sigma u_{x}^{2}(x, t)+\left(u_{x}+v_{x}\right)^{2}(x, t)\right) d x \leq \int_{\Omega}\left(\sigma u_{0, x}^{2}+\left(u_{0, x}+v_{0, x}\right)^{2}\right) d x .
$$

Since also

$$
u_{x}(., t) \rightarrow u_{0, x} \text { and } v_{x}(., t) \rightarrow v_{0, x} \text { weakly in } L^{2}(\Omega)
$$

as $t \rightarrow 0$, the assertion (2.27) follows.

Finally, the energy inequality (2.29) follows from (2.7) and the standard weakly lower semicontinuity argument. The proof of the theorem is complete. 


\section{Non-negativity of solutions}

In this section we prove that the global weak solutions constructed in the previous section are non-negative provided the initial data $\left(u_{0}, v_{0}\right)$ are non-negative. Furthermore, for the system (2.1) considered with intermolecular potentials $U_{1}(u), U_{2}(v)$, and $U_{3}(u+v)$ as in (1.5) we show existence of positive smooth solutions.

3.1. Non-negativity in the absence of intermolecular forces. Following the ideas of [25], we define a suitable entropy $G$ in order to show non-negativity of the weak solution $(u, v)$ from Theorem 2.1, provided (1.10) holds.

For $l \in\{2,3\}$ we set

$$
g_{\varepsilon, l}(s)=-\int_{s}^{A} \frac{d r}{\left(|r|^{l}+\varepsilon\right)^{1 / 2}}, G_{\varepsilon, l}(s)=-\int_{s}^{A} g_{\varepsilon, l}(r) d r,
$$

with a constant $A$ such that $A \geq \max \left\{\left|u_{\varepsilon}\right|,\left|v_{\varepsilon}\right|\right\}$ for all sufficiently small $\varepsilon$. Then one has

$$
G_{\varepsilon, l}^{\prime}(s)=g_{\varepsilon, l}(s), G_{\varepsilon, l}^{\prime \prime}(s)=g_{\varepsilon, l}^{\prime}(s)=\frac{1}{\left(|s|^{l}+\varepsilon\right)^{1 / 2}} .
$$

Also,

$$
g_{\varepsilon, l}(s) \leq 0, G_{\varepsilon, l}(s) \geq 0 \text { if } s \leq A
$$

and

$$
G_{\varepsilon, l}(s) \leq G_{0, l}(s) \text { for all } s \in \mathbb{R}^{1},
$$

where $G_{0, l}=\lim _{\varepsilon \rightarrow 0} G_{\varepsilon, l}$ such that for $0 \leq s \leq A$,

$$
G_{0, l}(s)=\left\{\begin{array}{lll}
\left(A-s-s \log \left(\frac{A}{s}\right)\right), & \text { if } & l=2, \\
2\left(\sqrt{A}+\sqrt{\frac{1}{A}} s-2 \sqrt{s}\right), & \text { if } & l=3 .
\end{array}\right.
$$

Since the structure of (1.2) is not symmetric with respect to $u$ and $v$ we use two entropies: one depending on $u$ and the other on $v$. For a fixed $\delta>0$ one has

$$
\begin{aligned}
& \quad \frac{d}{d t} \int_{\Omega}\left(G_{\varepsilon, 3}\left(u_{\varepsilon}\right)+G_{\varepsilon, 2}\left(v_{\varepsilon}\right)\right) d x=\int_{\Omega}\left(G_{\varepsilon, 3}^{\prime}\left(u_{\varepsilon}\right) u_{\varepsilon, t}+G_{\varepsilon, 2}^{\prime}\left(v_{\varepsilon}\right) v_{\varepsilon, t}\right) d x \\
& =\int_{\Omega}\left(G_{\varepsilon, 3}^{\prime \prime}\left(u_{\varepsilon}\right) u_{\varepsilon, x}\left(-\left(M_{11 \varepsilon}+\varepsilon\right) p_{1 \varepsilon, x}-M_{12 \varepsilon} p_{2 \varepsilon, x}\right)\right) d x \\
& \quad+\int_{\Omega}\left(G_{v \varepsilon}^{\prime \prime}\left(v_{\varepsilon}\right) v_{\varepsilon, x}\left(-M_{21 \varepsilon} p_{1 \varepsilon, x}-\left(M_{22 \varepsilon}+\varepsilon\right) p_{2 \varepsilon, x}\right)\right) d x \\
& \leq \frac{\delta}{2} \int_{\Omega}\left(( G _ { \varepsilon , 3 } ^ { \prime \prime } ( u _ { \varepsilon } ) ) ^ { 2 } ( M _ { 1 1 \varepsilon } + \varepsilon ) \left(\left(M_{11 \varepsilon}+\varepsilon\right) p_{1 \varepsilon, x}^{2}+2 M_{12 \varepsilon} p_{1 \varepsilon, x} p_{2 \varepsilon, x}\right.\right. \\
& \left.\left.\quad+\left(M_{22 \varepsilon}+\varepsilon\right) p_{2 \varepsilon, x}^{2}\right)\right) d x+\frac{\delta}{2} \int_{\Omega}\left(( G _ { v \varepsilon } ^ { \prime \prime } ( v _ { \varepsilon } ) ) ^ { 2 } ( M _ { 2 2 \varepsilon } + \varepsilon ) \left(\left(M_{11 \varepsilon}+\varepsilon\right) p_{1 \varepsilon, x}^{2}\right.\right. \\
& \left.\left.\quad+2 M_{12 \varepsilon} p_{1 \varepsilon, x} p_{2 \varepsilon, x}+\left(M_{22 \varepsilon}+\varepsilon\right) p_{2 \varepsilon, x}^{2}\right)\right) d x+\frac{1}{2 \delta} \int_{\Omega}\left(u_{\varepsilon, x}^{2}+v_{\varepsilon, x}^{2}\right) d x .
\end{aligned}
$$


By Definition 3.1 it follows that

$$
\left(G_{\varepsilon, 3}^{\prime \prime}\left(u_{\varepsilon}\right)\right)^{2}\left(M_{11 \varepsilon}+\varepsilon\right) \leq C \text { and }\left(G_{\varepsilon, 2}^{\prime \prime}\left(v_{\varepsilon}\right)\right)^{2}\left(M_{22 \varepsilon}+\varepsilon\right) \leq C,
$$

where in the last inequality we used the fact that there exists a constant $C$ such that $M_{22, \varepsilon} \leq C\left|v_{\varepsilon}\right|^{2}$ holds. Combining (3.4) with the energy inequality (2.7) and taking $0<\delta<1$ one obtains

$$
\begin{aligned}
& \frac{d}{d t} \int_{\Omega}\left(G_{\varepsilon, 3}\left(u_{\varepsilon}\right)+G_{\varepsilon, 2}\left(v_{\varepsilon}\right)\right) d x+\frac{d}{d t} E\left(u_{\varepsilon}, v_{\varepsilon}\right) \\
& \quad+(1-\delta) \int_{\Omega}\left(\left(M_{11 \varepsilon}+\varepsilon\right) p_{1 \varepsilon, x}^{2}+2 M_{12 \varepsilon} p_{1 \varepsilon, x} p_{2 \varepsilon, x}+\left(M_{22 \varepsilon}+\varepsilon\right) p_{2 \varepsilon, x}^{2}\right) d x \\
& \leq \frac{1}{2 \delta} \int_{\Omega}\left(u_{\varepsilon, x}^{2}+v_{\varepsilon, x}^{2}\right) d x \leq \frac{C}{2 \delta} \int_{\Omega}\left(\sigma u_{\varepsilon, x}^{2}+\left(u_{\varepsilon, x}+v_{\varepsilon, x}\right)^{2}\right) d x .
\end{aligned}
$$

This implies, using Gronwall', inequality, that

$$
\int_{\Omega}\left(G_{\varepsilon, 3}\left(u_{\varepsilon}\right)+G_{\varepsilon, 2}\left(v_{\varepsilon}\right)\right) d x \leq \exp \left(\frac{t}{\delta}\right) \int_{\Omega}\left(G_{\varepsilon, 3}\left(u_{0 \varepsilon}\right)+G_{\varepsilon, 2}\left(v_{0 \varepsilon}\right)\right) d x+E\left(u_{0 \varepsilon}, v_{0 \varepsilon}\right) .
$$

On the other hand by (1.10), (2.3), and (3.2-3.3) one has

$$
\begin{aligned}
\int_{\Omega}\left(G_{\varepsilon, 3}\left(u_{0 \varepsilon}\right)+G_{\varepsilon, 2}\left(v_{0 \varepsilon}\right)\right) d x & \leq \int_{\Omega}\left(G_{0,3}\left(u_{0 \varepsilon}\right)+G_{0,2}\left(v_{0 \varepsilon}\right)\right) d x \\
& \leq \int_{\Omega}\left(G_{0,3}\left(u_{0}\right)+G_{0,2}\left(v_{0}\right)\right) d x \leq C .
\end{aligned}
$$

Therefore, the last two estimates imply that for all $t \leq T$

$$
\int_{\Omega}\left(G_{\varepsilon, 3}\left(u_{\varepsilon}\right)+G_{\varepsilon, 2}\left(v_{\varepsilon}\right)\right) d x \leq C .
$$

Proceeding as in [25, Theorem 4.1] shows non-negativity of $u$ and $v$.

3.2. The case including intermolecular forces. In this section we consider the system (1.2)-(1.3) in the presence of the intermolecular forces given as in (1.5) considered with (1.9) and the initial data satisfying

$$
\begin{gathered}
u_{0}, v_{0}>0, \quad\left(u_{0}, v_{0}\right) \in H^{1}(\Omega) \times H^{1}(\Omega), \\
\int_{\Omega}\left(U_{1}\left(u_{0}\right)+U_{2}\left(v_{0}\right)+U_{3}\left(u_{0}+v_{0}\right)\right) d x \leq C_{1} .
\end{gathered}
$$

Theorem 3.1. Assume that $0<n<m$ and $m \geq 3$ in (1.5). Then a positive smooth solution to (1.2), (1.3), (1.5) coupled with (1.9), (3.10) exists for all $t \in(0, T)$.

Proof. Taking a suitable Hölder continuous regularization of $\Pi_{k}(s)$ and proceeding as in Section 2 one can show existence of regularized solutions $u_{\varepsilon}$ and $v_{\varepsilon}$ to $(2.2)$ 
considered now with (1.3), (1.5), (1.9), and (2.4) that satisfy the regularity properties as before. Note that in this case the energy inequality (2.7) for

$$
E\left(u_{\varepsilon}, v_{\varepsilon}\right)=\int_{\Omega}\left[\frac{\sigma}{2} u_{\varepsilon, x}^{2}+\frac{1}{2}\left(u_{\varepsilon, x}+v_{\varepsilon, x}\right)^{2}+U_{1}\left(u_{\varepsilon}\right)+U_{2}\left(v_{\varepsilon}\right)+U_{3}\left(u_{\varepsilon}+v_{\varepsilon}\right)\right] d x
$$

still holds. Therefore, using the fact that $U_{k}(s), k=1,2,3$ are bounded from below, and hence

$$
-\int_{\Omega}\left[U_{1}\left(u_{\varepsilon}\right)+U_{2}\left(v_{\varepsilon}\right)+U_{3}\left(u_{\varepsilon}+v_{\varepsilon}\right)\right] d x \leq C,
$$

together with (3.10) imply again the estimates (2.9)-(2.11).

We show additionally that there exists a constant $\delta$ independent of $\varepsilon$ such that

$$
u_{\varepsilon} \geq \delta>0, v_{\varepsilon} \geq \delta>0 \text { hold in } Q_{T} .
$$

Then proceeding to the limit $\varepsilon \rightarrow 0$ as in Theorem 2.1, the smoothness of the positive limits $u$ and $v$ will follow from the uniform parabolic theory and (3.12), and the statement of the theorem will be shown. Indeed, observe from (2.7) that

$$
\sup _{t \in(0, T)} \int_{\Omega}\left(U_{1}\left(u_{\varepsilon}(\cdot, t)\right)+U_{2}\left(v_{\varepsilon}(\cdot, t)\right)\right) d x \leq C .
$$

Since $U_{2}$ is bounded from below one has also

$$
\sup _{t \in(0, T)} \int_{\Omega} U_{1}\left(u_{\varepsilon}(\cdot, t)\right) d x \leq C .
$$

Let $u_{\varepsilon}\left(x_{0}, t\right)=\min _{\Omega} u_{\varepsilon}(\cdot, t)$. By Hölder continuity of $u_{\varepsilon}$ we get

$$
u_{\varepsilon}(x, t) \leq u_{\varepsilon}\left(x_{0}, t\right)+C\left|x-x_{0}\right|^{1 / 2} .
$$

Analogously to the proof for the single layer equation 1.1 in [1] one obtains for $0<$ $n<m$

$$
C \geq \int_{\Omega} U_{1}\left(u_{\varepsilon}(\cdot,, t)\right) d x \geq C_{2} \eta\left(u_{\varepsilon}\left(x_{0}, t\right)\right)+C_{3},
$$

where $\eta(s)=-\log s$ for $m=3, \eta(s)=s^{3-m}$ for $m>3$. Hence $u_{\varepsilon}\left(x_{0}, t\right)>0$ holds for all $t \in(0, T)$. The same argument works for $\min _{\Omega} v_{\varepsilon}(\cdot, t)$. Therefore (3.12) is true.

4. Existence of non-negative weak solutions in the intermediate-slip case

In this section, we show that the solution $\left(u_{\varepsilon}, v_{\varepsilon}\right)$ to the regularized system (2.2)(2.4) considered now with the intermediate-slip mobility matrix

$$
M=\left(\begin{array}{cc}
u^{2}+\varepsilon & u v \\
u v & (1+\beta) v^{2}+\varepsilon
\end{array}\right)
$$


converges to global non-negative weak solutions to (1.2) considered with (1.8), (2.1), and (1.9)-(1.10). Note that the case when intermolecular forces are present, i.e. for (1.2) considered with (1.4)-(1.5) and (1.8), proceeds then exactly as in Theorem 3.2 for the no-slip case.

The dissipation (2.7) of the energy functional (2.6) and the corresponding a priori estimates (2.9)-(2.13) and (2.17) are true in the intermediate-slip case as well. Therefore, (2.19) holds again up to a subsequence as $\varepsilon \rightarrow 0$. The following theorem states that the thus obtained limit $(u, v)$ is a non-negative global weak solution.

THEOREM 4.1. The pair $(u, v)$ satisfies for any $T>0$ the following properties:

$$
\begin{aligned}
& (u, v) \in C_{x, t}^{1 / 2,1 / 8}\left(\bar{Q}_{T}\right) \times C_{x, t}^{1 / 2,1 / 8}\left(\bar{Q}_{T}\right),(u, v) \in C_{x, t}^{4,1}(P) \times C_{x, t}^{4,1}(P), \\
& M_{11} p_{1, x}+M_{12} p_{2, x}, M_{21} p_{1, x}+M_{22} p_{2, x} \in L^{2}(P), \\
& u^{2} p_{1, x} \in L^{2}(R), v^{2} p_{2, x} \in L^{2}(S),
\end{aligned}
$$

where $P=\bar{Q}_{T} \backslash(\{u=0\} \cup\{v=0\} \cup\{t=0\}), R=\bar{Q}_{T} \cap\{v=0\} \cap\{|u|>0\}$, and $S=\bar{Q}_{T} \cap$ $\{u=0\} \cap\{|v|>0\}$. Furthermore, there exist functions $w_{1} \in L^{2}(R)$ and $w_{2} \in L^{2}(S)$ such that $(u, v)$ satisfies (1.2) in the following sense:

$$
\begin{aligned}
& \iint_{Q_{T}} u \phi_{t}-\iint_{P}\left(M_{11} p_{1, x}+M_{12} p_{2, x}\right) \phi_{x}-\iint_{R}\left(u^{2} p_{1, x}+u w_{1}\right) \phi_{x}=0, \\
& \iint_{Q_{T}} v \phi_{t}-\iint_{P}\left(M_{21} p_{1, x}+M_{22} p_{2, x}\right) \phi_{x}-\iint_{S}\left(v w_{2}+(1+\beta) v^{2} p_{2, x}\right) \phi_{x}=0,
\end{aligned}
$$

for all $\phi \in \operatorname{Lip}\left(\bar{Q}_{T}\right), \phi=0$ near $t=0$ and $t=T$,

$$
\begin{aligned}
& u(x, 0)=u_{0}(x), v(x, 0)=v_{0}(x), \quad x \in \bar{\Omega}, \\
& \int_{\Omega} u(x, t) d x=\left\|u_{0}\right\|_{L^{1}(\Omega)}, \int_{\Omega} v(x, t) d x=\left\|v_{0}\right\|_{L^{1}(\Omega)}, \\
& u_{x}(\cdot, t) \rightarrow u_{0, x}(\cdot) \text { and } v_{x}(\cdot, t) \rightarrow v_{0, x}(\cdot) \text { strongly in } L^{2}(\Omega) \text { as } t \rightarrow 0,
\end{aligned}
$$

and

$u$ and $v$ satisfy (1.9) at all points of the lateral

$$
\text { boundary, where } u \neq 0 \text { and } v \neq 0 \text {. }
$$

Finally, the following energy inequality holds:

$$
E(u(\cdot, T), v(\cdot, T))+\iint_{P}\left(M_{11} p_{1, x}^{2}+2 M_{12} p_{1, x} p_{2, x}+M_{22} p_{2, x}^{2}\right) \leq E\left(u_{0}, v_{0}\right) .
$$

Proof. The assertions (4.1)-(4.2) and (4.6)-(4.8) follow exactly as in the proof of Theorem 2.1. Using (2.10), one observes

$$
\begin{aligned}
\iint_{Q_{T}}\left(M_{11 \varepsilon} p_{1, \varepsilon, x}^{2}+M_{22 \varepsilon} p_{2 \varepsilon, x}^{2}\right) d x d t & \leq C-2 \iint_{Q_{T}} M_{12 \varepsilon} p_{1 \varepsilon, x} p_{2 \varepsilon, x} d x d t \\
& =C-2 \iint_{Q_{T}}\left(u_{\varepsilon} v_{\varepsilon} p_{1 \varepsilon, x} p_{2 \varepsilon, x}\right) d x d t .
\end{aligned}
$$


By Young's inequality,

$$
2 u_{\varepsilon} v_{\varepsilon} p_{1 \varepsilon, x} p_{2 \varepsilon, x} \leq \frac{2}{2+\beta} u_{\varepsilon}^{2} p_{1 \varepsilon, x}^{2}+\left(1+\frac{\beta}{2}\right) v_{\varepsilon}^{2} p_{2 \varepsilon, x}^{2},
$$

and hence one obtains

$$
\begin{array}{ll}
\iint_{Q_{T}} u_{\varepsilon}^{2} p_{1 \varepsilon, x}^{2} d x \leq C, & \iint_{Q_{T}} v_{\varepsilon}^{2} p_{2 \varepsilon, x}^{2} d x \leq C, \\
\iint_{Q_{T}}\left(M_{11 \varepsilon} p_{1, \varepsilon, x}^{2}+M_{22 \varepsilon} p_{2 \varepsilon, x}^{2}\right) d x d t \leq C .
\end{array}
$$

From the last inequality (4.10) then follows.

Next, for $\phi$ as in (4.5)-(4.5) one writes again (2.32)-(2.33). Considering the set $I_{1, \delta}$ and $I_{2, \delta}$ as in the proof of Theorem 2.1 and using the estimates (4.11) one can show in an analogous manner that (2.32) and (2.33) converge up to a diagonal subsequence as $\delta \rightarrow 0$ and $\varepsilon \rightarrow 0$ to (4.4) and (4.5) respectively.

Theorem 4.2. The global weak solution $(u, v)$ constructed in Theorem 4.1 is nonnegative.

Proof. The proof proceeds similarly to the argument for non-negativity of the weak solutions in the no-slip case presented in the Section 3.1. The estimates

$$
\left(G_{\varepsilon, 2}^{\prime \prime}\left(u_{\varepsilon}\right)\right)^{2}\left(M_{11 \varepsilon}+\varepsilon\right) \leq C, \quad\left(G_{\varepsilon, 2}^{\prime \prime}\left(v_{\varepsilon}\right)\right)^{2}\left(M_{22 \varepsilon}+\varepsilon\right) \leq C,
$$

and

$$
\begin{aligned}
& \frac{d}{d t} \int_{\Omega}\left(G_{\varepsilon, 3}\left(u_{\varepsilon}\right)+G_{\varepsilon, 2}\left(v_{\varepsilon}\right)\right) d x+\frac{d}{d t} E\left(u_{\varepsilon}, v_{\varepsilon}\right) \\
& \left.\quad+(1-\delta) \int_{\Omega}\left(M_{11 \varepsilon}+\varepsilon\right) p_{1 \varepsilon, x}^{2}+2 M_{12 \varepsilon} p_{1 \varepsilon, x} p_{2 \varepsilon, x}+\left(M_{22 \varepsilon}+\varepsilon\right) p_{2 \varepsilon, x}^{2}\right) d x \\
& \leq \frac{1}{2 \delta} \int_{\Omega}\left(u_{\varepsilon, x}^{2}+v_{\varepsilon, x}^{2}\right) d x,
\end{aligned}
$$

are used as analogues to (3.5)-(3.6) in this case in order to obtain the crucial estimate

$$
\int_{\Omega}\left(G_{\varepsilon, 2}\left(u_{\varepsilon}\right)+G_{\varepsilon, 2}\left(v_{\varepsilon}\right)\right) d x \leq C .
$$

\section{Conclusion and discussions}

In this article, we showed existence of non-negative global weak solutions for the coupled lubrication systems corresponding to the cases of no-slip and intermediateslip conditions at both liquid-liquid and liquid-solid interfaces. Our results can be generalized in a straight-forward way to the system (1.2) considered with the mobility matrix (1.6).

We should point out that the function $w$ appears in the weak formulation for the no-slip case (1.7) and is not identified in terms of the solution $(u, v)$. This is because 
of the strong coupling of the system (1.2) and consequently of the lack of suitable estimates on the singular set $R$. Furthermore, we emphasize that we obtained a slight difference between weak formulations in the no-slip and intermediate-slip cases (compare Theorem 2.1 with 4.1). Due to the fact that the components $M_{11}$ and $M_{22}$ in (1.8) depend only on $u$ or $v$, respectively, in contrast to the no-slip case there is no analogue of estimate (2.39) in the intermediate-slip case. Therefore, an additional (so far not identified) function $w_{2}$ appears on the singular set $S$ in the latter case. The same problem persists also in the weak-slip case as the leading orders of the mobility matrix components on the set $S$ coincide with those for the intermediate-slip case. In this sense we have "more regularity" for the weak solutions in the no-slip case than for the ones in the weak- or intermediate-slip cases. This interesting observation should be understood in more depth, in view of the fact that for the single lubrication equation 1.1 the no-slip case is known to be more singular from both the physical and the analytical points of view than the slip cases.

Another observation appearing as well due to different component structures of the mobility matrices (1.7) and (1.8) is that we have stronger entropy for $u$ than for $v$ in the case of the former matrix, whereas the entropies are the same for the latter one.

In contrast to the existing results for the single lubrication equation 1.1 (see e.g. $[25,26])$ we are not aware if the constructed weak solutions for the systems (1.2) should necessarily posses zero contact angles. This is due to an absence so far of the entropy dissipation inequality for the two layered systems (1.2) which was shown before to hold for (1.1). Combined energy-entropy dissipation inequalities derived here (as e.g. (3.6)) do not imply a priori $H^{2}$ estimates on the solutions. Similar difficulties with entropy estimates arose earlier, for example, in the proof of existence of non-negative weak solutions for a system modelling thin films on which surfactant molecules diffuse; see [30].

In previous investigations such as e.g. [15], the system (1.2) is often written in the variables $u$ and $u+v$ instead of $u$ and $v$. Such a transformation does not change the structure of (1.2). In particular, let $\bar{u}, \bar{v}$ be any linear transformation of $u, v$, e.g. $(\bar{u}, \bar{v})^{T}=A \cdot(u, v)^{T}$. Then, for symmetric and positive semi-definite matrix $M$, the system (1.2) becomes

$$
\begin{aligned}
\bar{u}_{t} & =\left(\bar{M}_{11} \bar{p}_{1, x}+\bar{M}_{12} \bar{p}_{2, x}\right)_{x}, \\
\bar{v}_{t} & =\left(\bar{M}_{12} \bar{p}_{1, x}+\bar{M}_{22} \bar{p}_{2, x}\right)_{x},
\end{aligned}
$$

where $\bar{p}_{1}=\delta E / \delta \bar{u}, \bar{p}_{2}=\delta E / \delta \bar{v}$, and the new mobility matrix $\bar{M}=A \cdot M \cdot A^{T}$ is symmetric and positive semi-definite as well. Our results in Section 2 are still valid. However, for the non-negativity result for $(u, v)$ (Section 3) our choice of variables seems to be the most convenient since in general $\bar{u}$ and $\bar{v}$ will not be non-negative.

In principle our proofs can be extended to lubrication systems with quite general mobility matrices that allow for positive definite approximations. But the identification of the flux on the singular sets (i.e. those where one of the liquid heights vanishes) will very strongly depend on the particular entries at the main diagonal of the mobility matrix, as has been observed for the two cases considered here. Note also that the non-negativity properties of the obtained weak solutions crucially depend on the concrete form of the main diagonal entries.

Recently, in $[31,32]$ it was shown that there are other thin film models which have similar gradient dynamics to the weak-slip two-layer model presented here, (1.2) with (1.6). For instance, thin film equations for a single layer which is covered by an 
insoluble surfactant [32]. In the latter model, the evolving variables denote the height of the thin film $h$ and the so called surface coverage $\tilde{\Gamma}$. In special cases, e.g. when the Lyapunov functional to these systems depends only on $h_{x}^{2}$ and $\left(\tilde{\Gamma}_{x}\right)^{2}$, our results will still apply.

Finally we note that when submitting our results, we have become aware of a different proof for the existence of weak solutions in the no-slip case in [29]. These authors used a different approach from ours for obtaining weak solutions, basing theirs on Galerkin approximations. But also in their weak formulation appear the functions $H_{f}$ and $H_{g}$ which are not identified on certain sets.

Acknowledgment. SJ is grateful for the support by the DFG of the project within the priority programme SPP 1506 "Transport at Fluidic Interfaces". The work of GK was supported by the postdoctoral scholarship at the Max-Planck-Institute for Mathematics in the Natural Sciences, Leipzig. SJ and GK would like to thank Andreas Münch, Dirk Peschka, and Barbara Wagner for fruitful discussions. The research of RT leading to these results has received funding from the European Community's Seventh Framework Programme FP7/2007-2013 under Grant Agreement no PIIFGA-2009-25452-TFE.

\section{REFERENCES}

[1] A.L. Bertozzi, G. Grün, and T.P. Witelski, Dewetting films: Bifurcations and concentrations, Nonlinearity, 14, 1569, 2001.

[2] M. Chugunova, M.C. Pugh, and R.M. Taranets, Nonnegative solutions for a long-wave unstable thin film equation with convection, SIAM J. Math. Anal., 42(4), 1826-1853, 2010.

[3] M. Chugunova and R.M. Taranets, Nonnegative Weak Solutions for a Degenerate System Modeling the Spreading of Surfactant on Thin Films, Applied Mathematics Research Express, 2012.

[4] V.S. Mitlin, Dewetting of solid surface: Analogy with spinodal decomposition, J. Colloid and Interface Sci., 156(2), 491-497, 1993.

[5] A. Münch, B. Wagner, and T.P. Witelski, Lubrication models with small to large slip lengths, J. Engin. Math., 53, 359-383, 2006.

[6] A. Oron, S.H. Davis, and S.G. Bankoff, Long-scale evolution of thin liquid films, Rev. Mod. Phys., 69(3), 931-980, 1997.

[7] G. Reiter, Dewetting of thin polymer films, Phys. Rev. Lett., 68(1), 75-78, 1992.

[8] R. Seemann, S. Herminghaus, and K. Jacobs, Dewetting patterns and molecular forces: $A$ reconciliation, Phys. Rev. Lett., 86(24), 5534-5537, 2001.

[9] U. Thiele, Thin film evolution equations from (evaporating) dewetting liquid layers to epitaxial growth, J. Phys.: Condensed Matter, 22(8), 084019, 2010.

[10] D. Bonn, J. Eggers, J. Indekeu, J. Meunier, and E. Rolley, Wetting and spreading, Rev. Mod. Phys., 81(2), 739, 2009.

[11] D. Bandyopadhyay, R. Gulabani, and A. Sharma, Instability and dynamics of thin liquid bilayers, Ind. Engin. Chem. Res., 44(5), 1259-1272, 2005.

[12] S. Jachalski, A. Münch, D. Peschka, and B. Wagner, Impact of interfacial slip on the stability of liquid two-Layer polymer films, J. Engin. Math., to appear.

[13] A. Pototsky, M. Bestehorn, D. Merkt, and U. Thiele, Morphology changes in the evolution of liquid two-layer films, J. Chem. phys., 122, 224711, 2005.

[14] L.S. Fisher and A.A. Golovin, Nonlinear stability analysis of a two-layer thin liquid film: Dewetting and autophobic behavior, J. Colloid and Interface Sci., 291(2), 515-528, 2005.

[15] A. Pototsky, M. Bestehorn, D. Merkt, and U. Thiele, Alternative pathways of dewetting for a thin liquid two-layer film, Phys. Rev. E, 70(2), 025201, 2004.

[16] L.S. Fisher and A.A. Golovin, Instability of a two-layer thin liquid film with surfactants: Dewetting waves, J. Colloid and Interface Sci., 307(1), 203-214, 2007.

[17] S. Jachalski, R. Huth, G. Kitavtsev, D. Peschka, and B. Wagner, Stationary solutions of liquid two-layer thin film models, SIAM J. Appl. Math., 73(3), 1183-1202, 2013.

[18] Z. Lin, T. Kerle, T.P. Russell, E. Schäffer, and U. Steiner, Electric field induced dewetting at polymer/polymer interfaces, Macromolecules, 35(16), 6255-6262, 2002. 
[19] H. Zeng, Y. Tian, B. Zhao, M. Tirrell, and J. Israelachvili, Friction at the liquid/liquid interface of two immiscible polymer films, Langmuir, 25(9), 4954-4964, 2009.

[20] R. Zhao and C.W. Macosko, Slip at polymer-polymer interfaces: Rheological measurements on coextruded multilayers, J. Rheology, 46(1), 145-167, 2002.

[21] N.P. Adhikari and J.L. Goveas, Effects of slip on the viscosity of polymer melts, J. Poly. Sci. Part B: Poly. Phys., 42(10), 1888-1904, 2004.

[22] J.L. Goveas and G.H. Fredrickson, Apparent slip at a polymer-polymer interface, Eur. Phys. J. B-Condensed Matter and Complex Systems, 2(1), 79-92, 1998.

[23] P.G. de Gennes, Wetting: Statics and dynamics, Rev. Mod. Phys., 57(3), 827-863, 1985.

[24] J.N. Israelachvili, Intermolecular and Surface Forces: Revised Third Edition, Academic Press, 2011.

[25] F. Bernis and A. Friedman, Higher order nonlinear degenerate parabolic equations, J. Diff. Equ., 83(1), 179-206, 1990.

[26] A.L. Bertozzi and M.C. Pugh, Long-wave instabilities and saturation in thin film equations, Commun. Pure Appl. Math., 51, 625-661, 1998.

[27] J.W. Barrett and L. El Alaoui, Finite element approximation of a two-layered liquid film in the presence of insoluble surfactants, ESAIM: Mathematical Modelling and Numerical Analysis, 42(05), 749-775, 2008.

[28] S.D. Eidel'man, Parabolic Systems, North Holland Pub. Co., 1969

[29] J. Escher and B.V. Matioc, Non-negative global weak solutions for a degenerated parabolic system approximating the two-phase Stokes problem, arXiv:1210.6457, preprint, 2012.

[30] H. Garcke and S. Wieland, Surfactant spreading on thin viscous films: Nonnegative solutions of a coupled degenerate system, SIAM J. Math. Anal., 37(6), 2025-2048, 2006.

[31] U. Thiele, Note on thin film equations for solutions and suspensions, Eur. Phys. J.-Special Topics, 197(1), 213-220, 2011.

[32] U. Thiele, A.J. Archer, and M. Plapp, Thermodynamically consistent description of the hydrodynamics of free surfaces covered by insoluble surfactants of high concentration, Phys. Flu., 24, 102107, 2012. 